\title{
ACTITUDES HACIA EL APRENDIZAJE COOPERATIVO Y ENFOQUE HISTÓRICO
}

\author{
Laura Haro-Pontón \\ Universidad Central del Ecuador, Quito, Ecuador \\ Iharo@uce.edu.ec \\ Lenin Reyes-Haro \\ Universidad de Barcelona, Barcelona, España \\ larhed84@yahoo.es \\ Benito León del Barco \\ Universidad de Extremadura, Extremadura, España \\ bleon@unex.es
}

Recepción Artículo: 18 enero 2020

Admisión Evaluación: 4 marzo 2020

Informe Evaluador 1: 13 marzo2020

Informe Evaluador 2: 18 marzo2020

Aprobación Publicación: 20 abril 2020

\section{RESUMEN}

La educación de la personalidad mediante procesos de enseñanza aprendizaje (PEA) sustentados en el enfoque histórico cultural, estimulan la zona de desarrollo próximo (ZDP) y promueven el desarrollo humano mediante particulares formas de organización de los individuos y del conocimiento. El desarrollo de sentimientos, actitudes, valores, pensamientos, imaginación, atención, entre otros, es su preocupación esencial. Este estudio tiene como objetivo demostrar que la enseñanza con el enfoque histórico cultural desarrolla actitudes favorables al trabajo en equipo. La investigación es de corte cuasi-experimental con un diseño pretest-postest con grupo control. Se seleccionaron para la intervención a estudiantes de los colegios con quienes trabajaron los docentes en formación en la práctica pre-profesional. Intervinieron en la investigación 835 estudiantes, 430 conforman el grupo experimental integrado por estudiantes de Básica Superior en una media de edad de $13,4( \pm 0,95)$ años y de Bachillerato de 16,4 $( \pm 1,6)$ años. El grupo control integran 405 estudiantes de Básica y Bachillerato, cuyas edades son similares al grupo experimental. En el PEA del grupo experimental se utilizó un diseño de clase que, en las formas organizativas pone énfasis en técnicas de trabajo cooperativo. Se aplicó el cuestionario de Predisposición y Actitudes ante el trabajo en equipo (CACTE). Tras la intervención, el grupo experimental tuvo un incremento significativo $(p<0,001)$ respecto al grupo control, su tamaño del efecto fue mediano $(r=0,27)$, situación que permite afirmar que trabajar en equipo con técnicas de aprendizaje cooperativo desarrolla sociabilidad, eleva la autoestima y garantiza aprendizajes significativos para la persona, aminora el individualismo y la competencia.

Palabras claves: enfoque histórico cultural; zona de desarrollo próximo (ZDP); aprendizaje cooperado; trabajo en equipo 


\section{ACTITUDES HACIA EL APRENDIZAJE COOPERATIVO Y ENFOQUE HISTÓRICO}

\section{ABSTRACT}

Attitudes towards cooperative learning and cultural historical psychology. Education of personality through teaching-learning processes (in Spanish, PEA) based on the cultural- historical psychology, stimulate the zone of proximal development (ZPD) and promote human development through particular forms of organization of individuals and knowledge. The development of feelings, attitudes, values, thoughts, imagination, attention among others, is its essential concern. That is why this study aims to demonstrate that teaching with a cultural-historical psychology develops a predisposition and attitudes towards teamwork. This study aims to demonstrate that teaching with a cultural- historical psychology, develops attitudes favourable to teamwork. The research is quasi-experimental with a pretest-posttest design and includes a control group. Students from schools with whom teachers worked in training in pre-professional practice were selected for intervention. 835 students participated in the research, from which 430 make up the experimental group consisting of students of Basic Superior in an average age of $13.4( \pm 0.95)$ years and Senior High School of $16.4( \pm 1.6)$ years. The control group includes 405 students of Basic education and Senior High school, whose ages are similar to the experimental group. In the PEA of the experimental group, a class design was used which, in organizational forms, emphasizes cooperative work techniques. The Predisposition and Attitudes towards Teamwork Questionnaire (in Spanish, CACTE) was applied. After the intervention, the experimental group had a significant increase $(p<0.001)$ with respect to the control group and its effect size was medium $(r=0.27)$, a situation that makes it possible to affirm that the application of teamwork with cooperative learning techniques, contributes to the development of sociability, raises self-esteem, and guarantees significant learning for the person, reduces individualism and competence.

Keywords: cultural historical approach; zone of proximal development (ZPD); cooperative learning; teamwork

\section{INTRODUCCIÓN}

El aprendizaje cooperativo, desde el enfoque histórico cultural, afirma que los estudiantes pueden aprender más con la ayuda de los demás que por sí solos, la interacción con los compañeros, los maestros, estimulan su (ZDP). El trabajo individual en las actividades educativas como única forma de aprender limita el aprendizaje de los estudiantes. Priorizar el trabajo en equipo, desplegar actividades cooperativas en el aula, es indispensable para desarrollar integralmente a los sujetos.

Este estudio analiza los elementos teóricos del enfoque histórico cultural y del aprendizaje cooperativo y los aplica en la investigación. Expone los resultados del trabajo en el aula con el enfoque mencionado, a través de actividades cooperadas que estimulan en los estudiantes su predisposición a aportar al desarrollo del conocimiento individual y colectivo, mediante su participación en equipos de trabajo.

Según el enfoque histórico cultural de Lev Vigotsky (1924) y sus discípulos, toda acción cultural es social. La cultura es producto de la vida y de la actividad social de los seres humanos (Vigotsky , 2000). Los individuos al nacer son seres sociales. La sociedad en las que les corresponde vivir, el medio donde se desarrollan, se encargan de acercarles a la cultura o marginarles, pero no pueden ignorar su condición de seres sociales. Las personas pueden existir al margen de la cultura, pero una sociedad que les niega su acceso, niega el derecho a ser considerados humanos (Fariñas, 2009) al impedirles que sean hombres y mujeres cultos, porque la educación enraíza a las personas a la cultura desde la infancia.

Desde este enfoque la educación es el elemento teórico y metodológico que propicia el desarrollo humano acorde a su realidad y necesidades de supervivencia, cultiva al ser social de forma sostenida. Vigotsky (2000) en la ley genética general del desarrollo cultural señala que toda función en el "... desarrollo cultural del niño aparece en escena dos veces en dos planos; primero en el plano social y después en el psicológico, al principio entre la gente como categoría interpsíquica y luego en el interior del niño como categoría intrapsíquica" (p. 150). La relación interpsíquica es la educativa. Esta ley genética se concreta en la ZDP, zona de interacción, construc- 
ción de conocimientos y enseñabilidad (González, 2008), porque al situarse en esta zona el PEA, garantiza que los estudiantes aprendan y se desarrollen.

El psiquismo humano es social, por la inserción de la persona en acciones culturales compartidas (Alessandroni, 2017). Todo niño con ayuda hace más que lo que puede por sí mismo, lo que realiza hoy en cooperación, en el futuro hará solo. Por ello, la educación que estimula y orienta se constituye en fuente de desarrollo (Vigotsky, 1990). Las actividades conjuntas socialmente positivas provocan dependencia responsable (Makarenko, 1977; Petrovski, 1989), Ios colectivos transmiten lo significativo para el grupo e incorporan características individuales de los compañeros. Esta interacción humana en el aprendizaje, mediadas por la palabra (Orrú, 2012) estimula la ZDP, informa, desarrolla afectos, establecen normas que regulan la participación de todos.

En otras latitudes diversas a la de Vigotsky, se cuestionaba también a la escuela tradicional, autoritaria y reproductiva, cuya respuesta surge en el Congreso de Calais (1921) donde proponen nuevos principios considerados válidos hasta hoy como: vinculación de la enseñanza con la vida, métodos activos y técnicas grupales, motivación. De esta propuesta surgen nuevos conceptos que se utilizan para referirse a las formas de aprender dentro de un grupo, tenemos así: aprendizaje grupal, aprendizaje colaborativo, cooperativo e interactivo.

En los años 70 irrumpe el concepto aprendizaje cooperado o cooperativo (AC), como acción colectiva para lograr objetivos. En EE.UU abordan el valor cognitivo de la interacción entre iguales mediante tutorías entre compañeros. Johnson y Johnson (1990) valoran el trabajo en grupos pequeños para garantizar unidad y un alto nivel de aprendizaje. El AC mejora la convivencia mediante relaciones positivas entre diversos grupos, provoca atracción interpersonal y mayor apoyo social (León, Polo, Gonzalo, Mendo, 2016). Para Coll (1984) el AC consolida y fortalece las relaciones de simpatía, cortesía, respeto, sentimientos de responsabilidad y ayuda mutua. El AC desarrolla intelectualmente al individuo, mediante la confrontación de puntos de vista, análisis y conclusiones. El aprendizaje colaborativo desarrolla a la persona por la acción del equipo (Reinoso 2005).

Investigadores como León, Mendo, Felipe, Polo, García, Gonzalo (2010-2017) analizan lo positivo de utilizar AC en el aula, para garantizar relaciones de calidad y compromiso en las interacciones interpersonales. Una actividad de AC debe garantizar la búsqueda y solución a problemas para ubicar las potencialidades del equipo; Ios grupos heterogéneos alcanzan mejores resultados. El AC es elemento integrador y formativo, cuando desarrolla habilidades sociales, comportamientos aprendidos, susceptibles de modificarse o afirmarse en los contextos de aprendizaje.

El PEA con enfoque histórico cultural apoyado en el AC además del desarrollo cognitivo de los estudiantes forma la personalidad de manera integral, fortalece sentimientos, actitudes, valores, desarrolla afecto por las acciones compartidas y aleja del individualismo y confrontación. El desarrollo de la actividad valorativa que aprecia los buenos resultados, los aportes creativos a la acción colectiva, muestra que la actitud es una construcción invisible pero latente (Mendo, Polo, Iglesias, Felipe. Y León, B., 2017)

El aprendizaje colaborativo se inicia con procesos graduales que se van desarrollando de forma individual en cada uno de los miembros del grupo comprometidos con el proceso de aprendizaje colectivo; se genera una interdependencia positiva por aprender con y de los otros. Este tipo de aprendizaje se adquiere mediante métodos de trabajo grupal que promueven habilidades, cuyo objetivo de aprendizaje es la interacción y el aporte de conocimientos de cada participante para crear nuevos aprendizajes.

Entre los beneficios de este modelo educativo están: el desarrollo personal en colectivo, habilidad para escuchar y ser crítico con las ideas de otros, reformular las opiniones de sus compañeros y reconstruir su pensamiento en procesos de análisis, centrarse en la toma de decisiones, reflexionar sus acciones y sus consecuencias, razón por la que pretendiendo obtener información sobre esta realidad, el principal objetivo de esta investigación fue evidenciar, que una enseñanza con el enfoque histórico cultural desarrolla la predisposición y actitudes ante el trabajo en equipo. Pero además se analizaron los contrastes y los cambios que se evidenciaron en los estudiantes con los que se trabajó en AC teniendo como base el enfoque histórico cultural y aquellos que no fueron objeto de la intervención. 


\section{ACTITUDES HACIA EL APRENDIZAJE COOPERATIVO Y ENFOQUE HISTÓRICO}

\section{MÉTODO}

\section{Participantes}

Conformaron la muestra 835 estudiantes de los colegios de Quito, en dos grupos de participantes, el primero denominado grupo experimental conformado por 430 estudiantes, seleccionados de los cursos donde practican docentes en formación de la Carrera de CCSS de la Universidad Central del Ecuador, 53 \% son mujeres y 47 \% son hombres, cuyas edades promedio de los 179 estudiantes de básica superior es de 13,4 $( \pm 0,95)$ años, y de 251 estudiantes de bachillerato es de 16,4 $( \pm 1,6)$ años. La muestra para el grupo control se tomó de colegios donde realizan las prácticas de observación y el (PEA) Io dirigen maestros de estas instituciones. Este grupo integraron 405 estudiantes, 42 \% son mujeres y 48 \% son hombres, cuyas edades de 303 estudiantes de básica superior es de $13( \pm 0,97)$ y de bachillerato de los 102 estudiantes es de 16,1 $( \pm 1,17)$. A los docentes en formación encargados de trabajar con el grupo experimental en el (PEAD) apoyados en TAC, se les capacitó durante el mes de septiembre y primero días del mes de octubre del 2016.

\section{Instrumentos}

El cuestionario de Actitudes hacia el Trabajo en Equipos de Aprendizaje (CACTE) es una escala que mide cómo el PEAD a través de trabajo de equipo potencia sentimientos de acción recíproca, desarrolla comportamientos de corresponsabilidad, infunde confianza en el colectivo y alcanza un buen nivel de seguridad individual. Consta de 12 items, que se puntúan en la escala de Likert de cinco intervalos, (desde 1 = Totalmente en desacuerdo, hasta 5 = Totalmente de acuerdo). La escala (CACTE) fue sometida a las pruebas de rigor necesarias, resultados que les permitió a los creadores del instrumento afirmar que su confiabilidad es buena y adecuada. El instrumento aplicado al grupo de intervención, ha evidenciado resultados necesarios para hacer una valoración más objetiva de cómo impactan las actividades cooperadas.

\section{Diseño}

La metodología es cuasi-experimental cuyo diseño es inter-grupo, pretest-postest, con grupo control; la incorporación de los integrantes a éstos no fue determinada de manera aleatoria, porque se respetó la forma natural en la que estuvieron organizadas las aulas. La metodología cuasi experimental presenta defectos que pueden atenuarse: a) incluyendo un grupo control y b) tomando medidas previo a la intervención y posterior a ella que se les denomina pre-test y pos-test (León, Mendo, Felipe, Polo y Fajardo, 2017).

Se realizó la medición pre-test de la variable dependiente (CACTE) en los dos grupos. La variable independiente (modelo de EA desde un enfoque desarrollador) se lo aplicó con posterioridad. Para identificar la consistencia de los cambios logrados luego de la intervención, la variable dependiente se midió de nuevo a los dos grupos.

\section{Procedimiento}

Se trabaja con profesionales de la educación en formación del sexto y séptimo nivel 2016-2017 que realizan la práctica preprofesional en diecisiete instituciones educativas de la ciudad en la aplicación del PEAD desde el enfoque histórico cultural apoyados en las TAC, luego que las autoridades de la Carrera de CCSS de la Universidad Central del Ecuador aprobaran la solicitud respectiva.

La capacitación a los profesionales de la educación en formación se desarrolló en septiembre y octubre del periodo 2016-2017, en TAC como: Técnica de Jigsaw o de Rompecabezas, Tutoría entre iguales, Cooperación guiada, Aprendiendo juntos y Grupos de Investigación.

El pre-test se ejecutó en la primera semana de actividades académicas de los colegios a los grupos experimental y control. La fase de intervención se desarrolló en 36 a 40 sesiones en las cuales se impartían asignaturas de CCSS, en las que se utilizaron las TAC. Se aplicaron las técnicas mencionadas en todos los cursos, en el bachillerato se dió mayor importancia a la técnica Grupos de investigación por ser más compleja y posibilitar 
variedad de experiencias de aprendizaje (León, Felipe, Iglesias y Marugán, 2014). En la fase final se administraron los cuestionarios (pos-test) a los dos grupos.

\section{Análisis de Datos}

El nivel de significación estadística para las pruebas fue de $p<0,05$ en este estudio. Para poder evaluar la normalidad de las distribuciones se utilizó la prueba de Kolmogorov-Smirnov y se demuestra que todas las variables tienen distribuciones no normales $(p<0,05)$. Se determinó así el uso de pruebas no paramétricas ( $U$ de MannWhitney y rangos con signos de Wilcoxon). La r (de Rosental) se utilizó para medir el tamaño del efecto.

Resultados

La variable "Predisposición y Actitudes ante el Trabajo en Equipo" (CACTE) mide la predisposición y actitudes ante el trabajo en equipo de los estudiantes. En la tabla 1 se muestran los estadísticos descriptivos (mediana, cuartiles y valores máximo y mínimo) de los factores de la escala CACTE, Factor 1: Actitudes Académicas y Factor 2: Actitudes Sociales y Afectivas, en versión experimental y control.

Tabla 1 Parámetros descriptivos de los factores de la escala CACTE-Estudiantes

\begin{tabular}{|c|c|c|c|c|c|c|c|c|c|c|}
\hline \multirow{2}{*}{$\begin{array}{c}\text { Intervenció } \\
\mathrm{n}\end{array}$} & \multicolumn{2}{|c|}{ Mínimo } & \multicolumn{2}{|c|}{ Máximo } & \multicolumn{2}{|c|}{ Mediana } & \multicolumn{2}{|c|}{ Cuartil 1} & \multicolumn{2}{|c|}{ Cuartil 3} \\
\hline & Pre & Post & Pre & Post & Pre & Post & Pre & Post & Pre & Post \\
\hline $\begin{array}{c}\text { Exp. act. } \\
\text { académicas }\end{array}$ & 10,00 & 8,00 & $\begin{array}{c}30,0 \\
0\end{array}$ & $\begin{array}{c}29,0 \\
0\end{array}$ & $\begin{array}{c}20,0 \\
0\end{array}$ & 21,00 & 18,00 & 20,00 & 22,00 & 23,00 \\
\hline $\begin{array}{c}\text { Exp. act. } \\
\text { soc. y } \\
\text { afect. }\end{array}$ & 6,00 & 8,00 & $\begin{array}{c}30,0 \\
0\end{array}$ & $\begin{array}{c}30,0 \\
0\end{array}$ & $\begin{array}{c}24,0 \\
0\end{array}$ & 25,00 & 21,00 & 22,00 & 27,00 & 28,00 \\
\hline $\begin{array}{l}\text { Cont. act. } \\
\text { académicas }\end{array}$ & 6,00 & 6,00 & $\begin{array}{c}53,0 \\
0\end{array}$ & $\begin{array}{c}48,0 \\
0\end{array}$ & $\begin{array}{c}20,0 \\
0\end{array}$ & 21,00 & 17,00 & 19,00 & 21,00 & 23,00 \\
\hline $\begin{array}{c}\text { Cont. acti. } \\
\text { soc. y } \\
\text { afect. }\end{array}$ & 6,00 & 6,00 & $\begin{array}{c}56,0 \\
0\end{array}$ & $\begin{array}{c}30,0 \\
0\end{array}$ & $\begin{array}{c}23,0 \\
0\end{array}$ & 24,00 & 20,00 & 21,00 & 25,00 & 27,00 \\
\hline $\begin{array}{c}\text { CACTE } \\
\text { exp. }\end{array}$ & 18,00 & $\begin{array}{c}18,0 \\
0\end{array}$ & $\begin{array}{c}56,0 \\
0\end{array}$ & $\begin{array}{c}58,0 \\
0\end{array}$ & $\begin{array}{c}44,0 \\
0\end{array}$ & $\begin{array}{c}46,50 \\
0\end{array}$ & 40,00 & 43,00 & 48,00 & 50,00 \\
\hline $\begin{array}{l}\text { CACTE } \\
\text { cont. }\end{array}$ & 12,00 & $\begin{array}{c}12,0 \\
0\end{array}$ & $\begin{array}{c}82,0 \\
0\end{array}$ & $\begin{array}{c}68,0 \\
0\end{array}$ & $\begin{array}{c}42,0 \\
0\end{array}$ & 45,00 & 38,00 & 40,00 & 46,00 & 49,00 \\
\hline
\end{tabular}

El factor 1 Actitudes Académicas según la tabla 2, no presenta diferencias en el momento pre $(p<0,074)$. En el momento post se manifiesta una mínima diferencia significativa $(p<0,017)$ aunque el tamaño del efecto es muy pequeño $(r=0,08)$. En el factor 2 Actitudes Sociales y Afectivas la diferencia es significativa $(p<0,001)$, sin embargo en los dos casos el tamaño del efecto es pequeño $(r=0,14)$. 


\section{ACTITUDES HACIA EL APRENDIZAJE COOPERATIVO Y ENFOQUE HISTÓRICO}

Tabla 2 Prueba U de Mann-Whitney para muestras independientes. Factores de la escala CACTE-Estudiantes

\begin{tabular}{|c|c|c|c|c|c|}
\hline & \multicolumn{2}{|c|}{ Mediana } & \multirow[b]{2}{*}{ Z } & \multirow[b]{2}{*}{$\begin{array}{c}\text { Sig. } \\
\text { (bilateral) }\end{array}$} & \multirow[b]{2}{*}{ Effect size $r$} \\
\hline & Experimental & Control & & & \\
\hline $\begin{array}{l}\text { Pre. act. } \\
\text { académicas }\end{array}$ & 20,00 & 20,00 & $-1,784$ &, 074 & $-0,06$ \\
\hline $\begin{array}{l}\text { Pre. act. soc. y } \\
\text { afectivas }\end{array}$ & 24,00 & 23,00 & $-4,333$ &, 000 & $-0,15$ \\
\hline $\begin{array}{l}\text { Post. act. } \\
\text { académicas }\end{array}$ & 21,00 & 21,00 & $-2,387$ & ,017 & $-0,08$ \\
\hline $\begin{array}{l}\text { Post. act. soc. } \\
\text { y afectiv. }\end{array}$ & 25,00 & 24,00 & $-3,927$ &, 000 & $-0,14$ \\
\hline
\end{tabular}

Los factores de la tabla 3 muestran después de la intervención un incremento en el grupo experimental, similar comportamiento se observa en el grupo control. Únicamente el factor 1, Actitudes Académicas, del grupo experimental tiene diferencia significativa $(p<0,001)$ y un efecto moderado $(r=0,25)$. El factor 2 , Actitudes Sociales y Afectivas, en el grupo experimental presenta diferencia significativa $(p<0,001)$ y un efecto limítrofe entre pequeño y moderado $(0,19)$. Los efectos en el grupo control de los dos factores son pequeños.

Tabla 3 Prueba de rangos con signos de Wilcoxon para muestras relacionadas. Factores de la escala CACTEEstudiantes

\begin{tabular}{|c|c|c|c|c|c|}
\hline & \multicolumn{2}{|c|}{ Mediana } & \multirow[b]{2}{*}{ Z } & \multirow[b]{2}{*}{$\begin{array}{c}\text { Sig. } \\
\text { (bilateral) }\end{array}$} & \multirow[b]{2}{*}{ Effect size $r$} \\
\hline & Pre & Post & & & \\
\hline $\begin{array}{c}\text { Exp. act. } \\
\text { académicas }\end{array}$ & 20,00 & 21,00 & $-7,302$ & ,000 & $-0,25$ \\
\hline $\begin{array}{l}\text { Exp. act. soc. y } \\
\text { afectivas }\end{array}$ & 24,00 & 25,00 & $-5,489$ &, 000 & $-0,19$ \\
\hline $\begin{array}{l}\text { Cont. act. } \\
\text { académicas }\end{array}$ & 20,00 & 21,00 & $-4,341$ & ,000 & $-0,15$ \\
\hline $\begin{array}{c}\text { Cont. act. soc. y } \\
\text { afectivas }\end{array}$ & 23,00 & 24,00 & $-3,691$ & ,000 & $-0,13$ \\
\hline
\end{tabular}

En la figura 1 se representan los resultados globales del instrumento CACTE, tanto en el grupo experimental como control. Las medianas de los pos-test son mayores que sus respectivos pre-test; sin embargo, los rangos intercuartílicos de las cuatro distribuciones se encuentran en un rango entre 40 y 50 puntos, lo que determina un solapamiento grande entre todas las dispersiones. 
Figura 1. Medianas, cuartiles y rangos intercuartílicos. Escala CACTE-Estudiantes

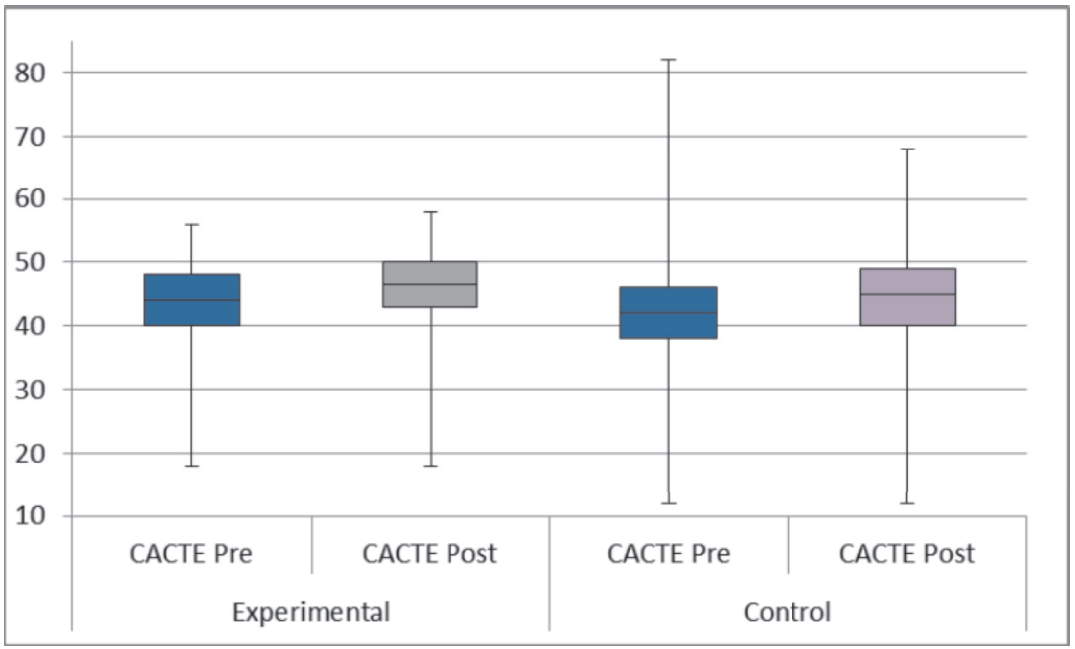

Si bien la comparación entre grupos en los momentos pre y pos-test, a través de la prueba $U$ de MannWhitney (tabla 4) muestra puntajes mayores en el grupo experimental de forma muy significativa $(p<0,001)$, el tamaño del efecto es pequeño $(r=0,12)$

Tabla 4

Prueba U de Mann-Whitney para muestras independientes. Escala CACTE-Estudiantes

\section{Mediana}

\begin{tabular}{cccccc}
\cline { 2 - 4 } & Experimental & Control & & $\begin{array}{c}\text { Sig. } \\
\text { (bilateral) }\end{array}$ & Effect size $\mathbf{r}$ \\
\hline CACTE Pre & 44,00 & 42,00 & $-3,580$ & $\mathbf{, 0 0 0}$ & $-0,12$ \\
CACTE Post & 46,50 & 45,00 & $-3,545$ & $\mathbf{, 0 0 0}$ & $-0,12$ \\
\hline
\end{tabular}

En el grupo experimental, el resultado pos-test es mayor que el pre-test de forma significativa $(p<0,001)$ y su tamaño del efecto es mediano. En el grupo control se replica la misma relación, donde el pos-test es mayor que el pre-test de forma significativa $(p<0,001)$; sin embargo, el tamaño del efecto es pequeño (Tabla 5).

Tabla 5 Prueba de rangos con signos de Wilcoxon para muestras relacionadas. Escala CACTE-Estudiantes

\section{Mediana}

\begin{tabular}{|c|c|c|c|c|c|}
\hline & Pre & Post & Z & $\begin{array}{c}\text { Sig. } \\
\text { (bilateral) }\end{array}$ & Effect size $r$ \\
\hline CAPTE Exp & 44,00 & 46,500 & $-7,804$ &, 000 & $-0,266114191$ \\
\hline CAPTE Control & 42,00 & 45,00 & $-4,692$ &, 000 & $-0,16486008$ \\
\hline
\end{tabular}

\section{DISCUSIÓN}

Este estudio pretende demostrar que un PEAD, con enfoque histórico cultural, potencia la predisposición a trabajar en equipo. 


\section{ACTITUDES HACIA EL APRENDIZAJE COOPERATIVO Y ENFOQUE HISTÓRICO}

Se partió desde el enfoque histórico cultural que concibe al entorno social como condición intrínseca que favorece u obstaculiza el desarrollo de la psiquis humana y las funciones psíquicas superiores se originan en las relaciones humanas, que van de lo social, lo externo a lo individual e interno (Vigotsk, 1979). Las interacciones educativas que se desarrollan dentro o fuera del aula, estimulan la ZDP mediante procesos colaborativos que facilitan la experimentación, la construcción del conocimiento, desarrollan destrezas, capacidades, que redunden en la formación de sentimientos, valores, convicciones e ideales. Este marco permite interpretar los resultados obtenidos en la investigación de factores de CACTE en muestras independientes como son: Actitudes académicas y Actitudes sociales y afectivas.

Respecto al factor Actitudes Académicas, algunos investigadores consideran que el aprendizaje cooperativo promueve un mejor rendimiento y productividad de quienes integran el equipo de trabajo (Johnson, Maryuama, Johnson, Nelson y Skon, 1981). En este estudio la diferencia que se presenta antes de la intervención y posterior a ella es pequeña, situación que evidencia que el nivel de involucramiento, compromiso, coparticipación, no es asumida por todos los miembros del equipo de trabajo. La interdependencia fortalece la cohesión y confianza mutua, la dependencia de unos y otros garantiza el logro de determinados propósitos. El factor actitudes sociales y afectivas expresa una diferencia significativa entre el grupo experimental y control en el momento pre, mientras que en el momento post se mantiene una diferencia similar a la obtenida antes de la intervención debido a un incremento similar de las puntuaciones en los dos grupos. En estos dos últimos casos el tamaño del efecto es pequeño, resultado que permite entender que, la coparticipación no es aún el elemento que fortalece sentimientos de respeto, admiración, afecto y confianza en los integrantes del equipo.

Los estudiantes del grupo experimental y control mostraron un incremento significativo de actitudes académicas y sociales/afectivas tras la intervención. Sin embargo, solo las actitudes académicas del grupo experimental mostraron un tamaño del efecto moderado. Las actitudes sociales y afectivas del mismo grupo experimental estuvieron en el límite entre pequeño y moderado. En el grupo control todos los efectos fueron pequeños.

En los últimos tiempos en las instituciones educativas hay un manifiesto interés por incorporar actividades grupales en el aula. Estas se han implementado de manera un tanto ingenua por docentes poco capacitados en el manejo de técnicas de aprendizaje cooperado. Ignoran que en las actividades de coparticipación, intervienen las ideas o concepciones que el equipo maneja para realizar con éxito una tarea emprendida (León et al., 2017). Desconocer que mediante acciones cooperadas se aprende y se desarrollan cualidades humanas, merma la confianza respecto al aporte responsable de los otros, situación que induce a pensar aún a un 25 \% del grupo experimental que más se aprende solo que en equipos de trabajo.

Los resultados manifiestan que en la mayoría de los educandos que fueron intervenidos con técnicas cooperadas en el PEAD con enfoque histórico cultural, mejoró la predisposición para trabajar en equipo. Reconocen que al ser parte de un grupo y trabajar con responsabilidad dentro de él, logran identificar en menor tiempo la solución a los problemas. Consideran que la participación colectiva mejora la calidad de los trabajos, desarrolla sociabilidad, eleva la autoestima y construye relaciones de amistad duraderas al mismo tiempo que aprenden.

De acuerdo a los resultados alcanzados en los factores (CACTE) del grupo experimental en relación al grupo control las mejoras son moderadas, considerando el tamaño del efecto. Tómese en cuenta que en el campo educativo, según Valentine y Cooper (2003), las investigaciones pueden presentar valores más bajos que en otras disciplinas cuando se experimenta con nuevas metodologías; por ejemplo, valores como $d=30$ y $d=33$ aunque no se manifiesten diferencias significativas, son estimados relevantes (Mendo et al., 2016, p.150). En los resultados de este estudio, el tamaño del efecto intragrupo (pretest- postest) manifiesta que los logros en el PEA en la variable CACTE es pequeña.

En los resultados intergrupo (experimental-control) los estudiantes del grupo experimental mostraron en su actitud para trabajar en equipo un incremento significativo luego de la intervención, esto se evidencia cuando el pos-test es mayor que el pre-test de forma significativa y el tamaño del efecto es mediano. En el grupo control se manifiestan también estos resultados, realidad que permite entender que el grupo control no estuvo al margen de 
la acción educativa, cuando manifiesta que el pos-test es mayor que el pre-test de forma significativa, aunque el tamaño del efecto es pequeño.

La dirección del PEAD la tuvieron profesionales de la educación en formación, maestros nóveles, cuyos resultados se los puede considerar positivos. Pero pueden superarse, si la dirección de estas acciones las realizan los docentes de las instituciones educativas, con previa capacitación en el manejo de técnicas de trabajo cooperadas que contribuyan al desarrollo y formación integral de los educandos.

El cuestionario CACTE, que se utilizó en esta investigación, fue diseñado para poblaciones universitarias (Mendo et al., 2017), pero los resultados evidenciaron que el instrumento sí permite obtener la información en muestras de estudiantes de colegio de Básica y Bachillerato, información obtenida que expone una realidad educativa en su contexto natural, sin que medie ningún artificio.

Futuras investigaciones deberán realizarse con maestros titulares de las asignaturas capacitados en técnicas de aprendizaje cooperativo TAC y cómo éstas amplían la ZDP de los estudiantes.

Educar en la solidaridad, respeto y cooperación debe ser un compromiso de las instituciones educativas en el momento actual, frente a escenarios caóticos, de confrontación, agresión, irrespeto, aprovechamiento del más débil y hasta de muerte. El profesorado, las instituciones educativas deben constituirse en gestores de ambientes propicios donde además del aprendizaje científico-técnico, se practique la paz, solidaridad, fraternidad y respeto mutuo.

\section{REFERENCIAS BIBLIOGRÁFICAS}

Alessandroni, N. (2017). Imaginación, creatividad y fantasía en Lev S. Vigotsky: una aproximación a su enfoque sociocultural. Actualidades en Psicología, 13 (51-52), 41-77.

Coll, C. (1984). Estructura grupal, interacción entre alumnos y aprendizaje escolar. Universidad de Barcelona. Infancia y aprendizaje , 27(28), 119-138

Fariñas, G. (2009). El enfoque histórico cultural en el estudio del desarrollo humano: para una praxis humanista. Revista Electrónica "Actualidades Investigativas en Educación", Vol 9 1-23.

González, A. (2008). Modelo Didáctico para el diseño de situaciones de enseñanza aprendizaje desarrolladora en la formación inicial del Profesor General Integral de Secundaria Básica. La Habana: "EJV".

Johnson, D. W. (1981). Effects of cooperative, competitive and individualistic goal structures on achievement. A meta-analysis. Psychological Bulletin, 89, 47-62. doi: 10.1037/0033-2909.89.1.47.

Johnson, D. y. (1990). Cooperation and competition theory and research. Addison-Wesley: Hillsdale, N.J.

León, B., Felipe, E., Iglesias, D. y Marugán, M (2014). Determinantes en la eficacia del aprendizaje cooperativo. Una experiencia en el EEES. Revista de Investigación Educativa, 32(2), 411-424.

León, B., Polo, M., Gozalo, M., Mendo, S. (2016). Relevancia del aprendizaje cooperativo sobre los diferentes perfiles de la dinámica bullying. Un análisis mediante pruebas de tamaño del efecto. Anales de Psicología, 32(1), 80-88. Recuperado de: https://doi.org/10.6018/analesps.32.1.183141

León, B., Mendo, S., Felipe, E., Polo, M., Fajardo F. (2017). Potencia de equipo y aprendizaje cooperativo en el ambito universitario. Revista de Psicodidáctica, 22(1). 9-15.

McGuirre, W., Kiesler, S., y Siegel, J. (1987). Group and computer - mediated discussion effects in risk decision. Making. Jounal of Personality and social Psyckology, 52.917-930.

Makarenko, A. (1977). La colectividad y la educación de la personalidad. La Habana: Editorial Pueblo y Eduación.

Mendo, S., León, B., Felipe, E., Polo, M., y Palacios, V. (2016). Evaluación de las habilidades sociales de estudiantes de Educación Social. Psicodidáctica, 21 (1),139 - 156. Recuperado de: https://doi.org/10.1387/RevPsicodidact.14031

Mendo, S., Polo, M., Iglesias, D., Felipe, E., y León, B. (1917). Construction and Validation of a Measurement Instrument for Attitudes towards Teamwork. Frontier in Psichology , 8. 6. Recuperado de: 10.3389/fpsyg.2017.01009. 


\section{ACTITUDES HACIA EL APRENDIZAJE COOPERATIVO Y ENFOQUE HISTÓRICO}

Orrú, S. (2012). Bases conceptuales del enfoque histórico-cultural para la comprensión del lenguaje. Estudios Pedagógicos, 38(2), 337-353. Recuperado de: http://www.scielo.cl/pdt/estped/v38n2/art21.pdf.

Petrovski, A. (1989). Teoría Psicológica del Colectivo. La Habana: Editorial Pueblo y Educación.

Reinoso, C. (2005). Un modelo pedagógico para contribuir al desarrollo de la actividad pedagógica cooperada. La Habana: Instituto Central de Ciencias Pedagógicas.

Valentine, J. y. (2003). Effect Size Substantive Interpretation Guidelines: Issues in the Interpretation of Effect Sizes. Washington. D. C.: What Works Clearing House.

Vigotsky, L. (1979). El desarrollo de los procesos psicológicos superiores. Barcelona: Grijalbo.

Vigotsky, L. (1990). Pensamiento y Lenguaje. Madrid: Centro de publicaciones del M.E.C.

Vigotsky, L. (2000). Historia de las funciones psíqicas superiores. Madrid: Visor Dis. 